\section{CRISPR/Cas9-mediated knockin of human factor IX into swine factor IX locus effectively alleviates bleeding in hemophilia B pigs}

\author{
Jiahuan Chen, ${ }^{1 *}$ Beiying An, ${ }^{2 *}$ Biao Yu, ${ }^{1}$ Xiaohuan Peng, ${ }^{1}$ Hongming Yuan, ${ }^{1}$ \\ Qiangbing Yang, ${ }^{1}$ Xue Chen, ${ }^{1}$ Tingting Yu, ${ }^{1}$ Lingyu Wang, ${ }^{1}$ Xinwei Zhang, ${ }^{1} \mathrm{He}$ \\ Wang, ${ }^{1}$ Xiaodong Zou, ${ }^{1}$ Daxin Pang, ${ }^{1}$ Hongsheng Ouyang ${ }^{1}$ and Xiaochun Tang ${ }^{1}$
}

${ }^{1}$ College of Animal Sciences, Jilin University, Changchun and ${ }^{2}$ Department of Medical Laboratory, The First Hospital of Jilin University, Changchun, China

*JC and BA contributed equally as co-first authors.

\section{ABSTRACT}

$\mathrm{H}$ emophilia B is an X-linked recessive bleeding disorder caused by abnormalities in the coagulation factor IX gene. Without prophylactic treatment, patients experience frequent spontaneous bleeding episodes. Well-characterized animal models are valuable for determining the pathobiology of the disease and for testing novel therapeutic innovations. Here, we generated a porcine model of hemophilia $\mathrm{B}(\mathrm{HB})$ using a combination of CRISPR/Cas9 and somatic cell nuclear transfer. We also tested the possibility of HB therapy by gene insertion. Frequent spontaneous joint bleeding episodes that occurred in $\mathrm{HB}$ pigs allowed a thorough investigation of the pathological process of hemophilic arthropathy. In contrast to the HB pigs, which showed a severe bleeding tendency and joint damage, the transgenic pigs carrying human coagulation factor IX exhibited a partial improvement in bleeding. In summary, this study not only offers a translational $\mathrm{HB}$ model for exploring the pathological process of hemophilic arthropathy, but also provides a possibility for the permanent correction of hemophilia in the future by genome editing in situ.

\section{Introduction}

Factor IX (FIX) is a vitamin K-dependent plasma protein that participates in the intrinsic blood coagulation pathway by converting factor $\mathrm{X}$ to its active form in the presence of $\mathrm{Ca}^{2+}$, phospholipids, and factor VIIIa. ${ }^{1}$ FIX is primarily synthesized in the liver and secreted into plasma. Defects in F9 will cause hemophilia B (HB), which is an inherited X-linked bleeding disorder. Based on the residual FIX plasma levels, the disease is classified as mild $(5-40 \%)$, moderate $(1-5 \%)$, and severe $(<1 \%){ }^{2}$ The morbidity of $\mathrm{HB}$ is $1: 25,000$ male live births, and according to the latest global report from the World Federation of Hemophilia there are 28,775 patients worldwide with $\mathrm{HB}^{3,4}$ Recurrent spontaneous bleeding mainly occurs in the synovial joints, particularly in the knees, ankles, and elbows. ${ }^{5}$ These bleeds may lead to chronic pain, immobility and a significant reduction in quality of life. This debilitating condition is the most frequent complication of hemophilia, known as hemophilic arthropathy. ${ }^{6}$

The current management for $\mathrm{HB}$ requires lifelong intravenous protein replacement therapy with FIX, which is costly, inconvenient and not curative. HB, which is caused by a single gene abnormality, is an attractive target for gene therapy. Gene therapy using adeno-associated viral (AAV) vectors has shown successful amelioration of severe bleeding phenotypes in animal experiments and clinical trials. ${ }^{711}$ However, because of the non-integrating nature of the AAV vector and the high hepatocyte cellular turnover, in some cases the expression of FIX decreases over time. ${ }^{11,12}$ Genome editing can allow stable transgene expression by site-specific gene integration, and the CRISPR/Cas9 gene editing tool shows promise for efficient correction of monogenic diseases. ${ }^{13}$

Animal models of hemophilia and related diseases are important for evaluating novel therapeutic strategies and understanding some pathological processes that are
Haematologica 2021

Volume 106(3):829-837

\section{Correspondence: \\ XIAOCHUN TANG \\ xiaochuntang@jlu.edu.cn}

Received: April 11, 2019.

Accepted: January 22, 2020.

Pre-published: January 23, 2020.

https://doi.org/10.3324/haematol.2019.224063

(C)2021 Ferrata Storti Foundation

Material published in Haematologica is covered by copyright. All rights are reserved to the Ferrata Storti Foundation. Use of published material is allowed under the following terms and conditions:

https://creativecommons.org/licenses/by-nc/4.0/legalcode. Copies of published material are allowed for personal or internal use. Sharing published material for non-commercial purposes is subject to the following conditions: https://creativecommons.org/licenses/by-nc/4.0/legalcode, sect. 3. Reproducing and sharing published material for commercial purposes is not allowed without permission in writing from the publisher. 
not completely clear. ${ }^{14,15}$ No single animal model responds to all the requirements for studying every aspect of a disease. Classical hemophilic mice and dogs have greatly accelerated the exploration of the pathophysiology and treatment of hemophilia. ${ }^{14-16}$ The advantages of hemophilic mice are availability, fast reproduction, and easy handling. They have proved to be instrumental in screening the pharmacokinetic and pharmacodynamic properties of potential drugs and gene therapeutics prior to other animal models of hemophilia. ${ }^{17,18}$ However, studies with hemophilic mice allow only small-volume blood samples and a limited frequency of blood sampling. Moreover, spontaneous bleeding rarely occurs in hemophilic mice, and intervention is necessary in certain studies. ${ }^{16,19,20}$ For decades, therapeutic efficacy in hemophilic dogs has proven to be an excellent predictor of human clinical efficacy. ${ }^{16}$ In addition, hemophilic dogs are frequently used to validate the effects of gene therapy. ${ }^{21-23}$ They show the spontaneous bleeding phenotype; however, the relatively infrequent occurrence of clinically recognizable spontaneous joint bleeding currently hampers any in-depth study of hemophilic arthropathy. ${ }^{16}$ Pigs are an excellent animal model for understanding the pathogenesis of human disease and developing therapeutic strategies. ${ }^{24-26}$ They are similar to humans in anatomy, physiology, and genome. Therefore, we decided to generate $\mathrm{HB}$ pigs that potentially provide their own unique study opportunities. Moreover, CRISPR/Cas9-mediated homologydirected gene targeting was used to determine whether it could ameliorate the bleeding phenotype by site-specific gene insertion.

\section{Methods}

\section{Animal studies and ethics statements}

All animal studies and the breeding process were carried out in accordance with guidelines approved by the Animal Welfare and Research Ethics Committee of Jilin University. All invasive procedures were performed under inhalation anesthesia with 1.5\% isoflurane. The wild-type (WT) controls used in our study were age- and sex-matched.

\section{Plasmid construction}

pX330-U6-Chimeric_BB-CBh-hSpCas9 was a gift from Feng Zhang (Addgene plasmid \#42230). ${ }^{27}$ Two paired oligonucleotides, which were designed on the basis of porcine F9 sequences, were ligated to the pX330 plasmid to form single guide RNA (sgRNA)expressing vectors. The coding sequences (CDS) of human F9, two homologous DNA arms of porcine F9 sequences and the pLB vector (TIANGEN, VT205) were assembled together to generate the donor vector. Details of the methods used are available in the Online Supplementary Methods.

\section{Cell culture and selection}

The constructed sgRNA-expressing vectors and the donor vector were transfected via electroporation into cultured porcine fetal fibroblasts (PFF). Fibroblasts were isolated from 33-day old fetuses of Large White pigs. The transfected cells were cultured in Dulbecco's modified Eagle's medium (Gibco, USA) supplemented with $15 \%$ fetal bovine serum (Gibco) at $39^{\circ} \mathrm{C}$ in an incubator with $5 \% \mathrm{CO}_{2}$. The positive PFF were selected by the limiting dilution method. Details are given in the Online Supplementary Methods.

\section{Somatic cell nuclear transfer and embryo transfer}

The positive cells were pooled as donor cells for somatic cell nuclear transfer (SCNT) ${ }^{28}$ Briefly, a single donor cell was microinjected into an enucleated pig oocyte. Then, the reconstructed embryos were activated and transferred into the synchronized recipient pigs. We performed ultrasonography 30 days post transfer and obtained cloned pigs by eutocia.

\section{Quantitative real-time polymerase chain reaction and western blot analysis}

Standard protocols were used for RNA and protein isolation, quantitative real-time polymerase chain reaction (qRT-PCR) and western blot analysis. Details are given in the Online Supplementary Methods.

\section{Clinical observations}

An 8-week clinical observational recording process was initiated after the piglets were born. The pigs were examined daily, and the number and type of bleeding episodes were observed, with a particular focus on joints.

\section{Blood sampling and blood analysis}

A detailed description of the blood sampling and blood analysis is provided in the Online Supplementary Methods.

\section{Radiography}

Radiographs of the pig limbs were obtained using a digital Xray imaging system (PaxScan 4343R, Varian; Palo Alto, CA, USA).

\section{Histological assessment}

The ankle joints and livers from 2-month old pigs were placed in $4 \%$ formaldehyde for fixation. The joints were decalcified for 3 weeks before tissue processing and paraffin embedding. The liver sections were stained with Hematoxylin \& Eosin (HE). The ankle joints were stained with $\mathrm{HE}$ and Safranin $\mathrm{O}$. After examination by light microscopy, HE-stained sections were scored for the presence of synovitis, and Safranin $\bigcirc$ stains were scored for cartilage degradation. The score was based on a modified grading system of hemophilic synovitis; ${ }^{29}$ scoring criteria are shown in Online Supplementary Table S1.

\section{Statistical analysis}

The data from the experiments were analyzed with GraphPad Prism software ( $t$-test). $P<0.05$ was considered statistically significant.

\section{Results}

\section{Generation of porcine F9 knockout pigs and human F9 knockin pigs}

Two sgRNAs were specifically designed to target the porcine F9 gene using CRISPR/Cas9 (Figure 1A), considering the following: (i) a computational BLAST tool was used to analyze the selected sgRNAs to ensure that they are unique in the porcine genome, reducing the risk of offtarget gene editing; (ii) to produce a non-functional coagulation factor IX protein, the target sites were selected at the 5' end of the F9 gene; and (iii) the target sites contained mutations known to cause $\mathrm{HB}$, based on the human FIX database (http://www. factorix.org). We transfected male and female fetal pig fibroblasts with the two Cas9/sgRNA plasmids separately, 118 fetal pig fibroblasts (88 males; 30 females) were screened by PCR and we identified 13 positive cell clones that contained 11 male clones and two female clones (Online Supplementary Figure S1). We selected some positive clones for SCNT, and the 
reconstructed embryos were transferred into nine surrogate pigs (Online Supplementary Table S2). Ultrasonography was performed 30 days post transfer and revealed that four recipients had become pregnant. All pregnancies were maintained to term, and 12 founder (F0) pigs (8 males; 4 females) were delivered. Genotyping by PCR and DNA sequencing identified that eight male pigs carried an F9 deletion and four female pigs had a heterozygous genotype (Figure $1 \mathrm{~B}$ and $\mathrm{C}$ ). The female $\mathrm{F} 0$ pigs were used to mate with the male F0 pigs to generate F1 pigs. The breeding gave rise to seven F1 pigs, including two male WT pigs, two male F9-deletion pigs, and three

A

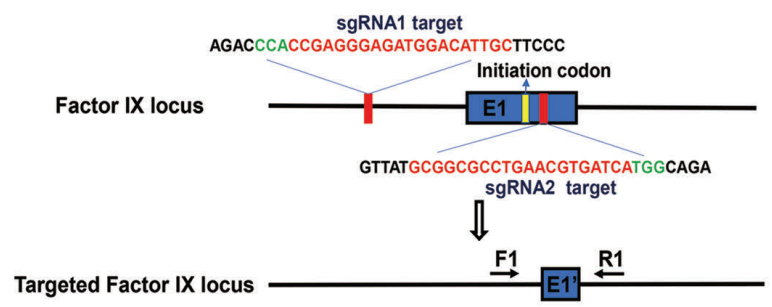

female heterozygous pigs (Online Supplementary Figure S2)

Meanwhile, we wondered whether the insertion of a single copy of the human Fq ( $h F 9$ ) gene could alleviate the phenotype in porcine $F 9(\mathrm{pF} q)$ knockout $(\mathrm{KO})$ pigs. Thus, in addition to the previously identified two sgRNAs, a donor vector that carries the hFg CDS flanked by $1-\mathrm{kb}$ homology sequences on both sides was also generated (Figure 1D). By co-transfecting the Cas9/sgRNA plasmids and the donor vector into male fetal pig fibroblasts, we obtained five positive cell clones, and the subsequent sequencing results showed that the $h F 9$ CDS was inserted correctly into the pFg locus (Online Supplementary Figure
B

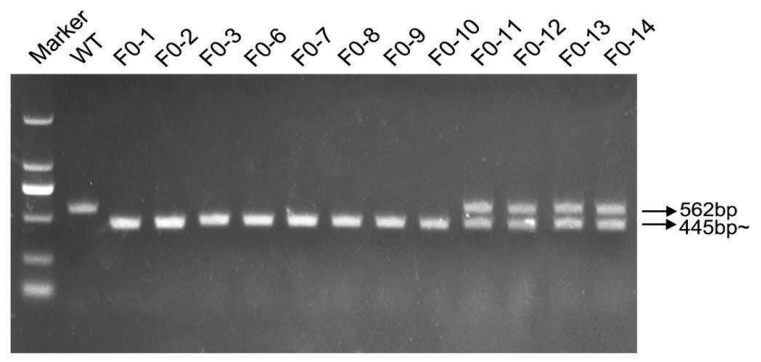

C

WT AAGACCCACCGAGGGAGATGGACATTGCTTC----76bp----GTTATGCGGCGCCTGAACGTGATCATGGCAGAAT

KO-T1 AAGACCCACCGA--117bp

KO-T2 AAGACCCACCG -

WT AAGACCCACCGAGGGAGATGGACATTGCTTC----76bp----GTTATGCGGCGCCTGAACGTGATCATGGCAGAAT AAGACCCACCGAGGGAGATGGACATTGCTTC----76bp----GTTATGCGGCGCCTGAACGTGATCATGGCAGAAT

KO-T1 AAGACCCACCGAGGGAGATGGACATTGCTTC----76bp----GTTATGCGGCGCCTGAACGTGATCATGGCAGAAT AAGACCCACCGA-
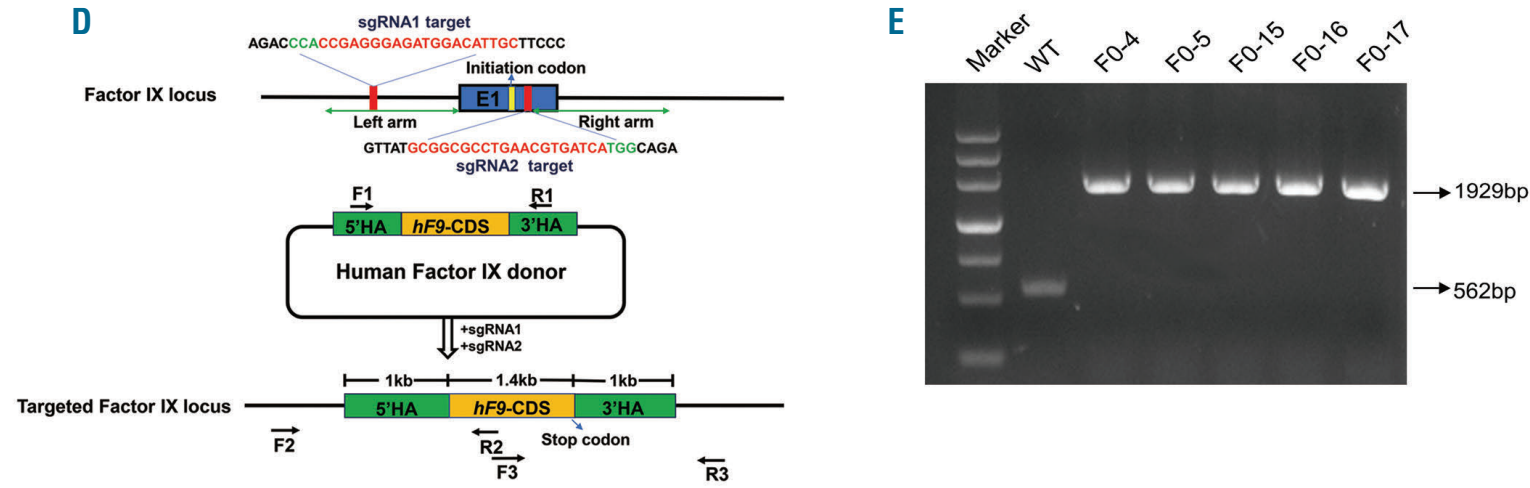

F

WT AAGACCCACCGAGGGAGATGGACATTGCTTC----76bp----GTTATGCGGCGCCTGAACGTGATCATGGCAGAAT

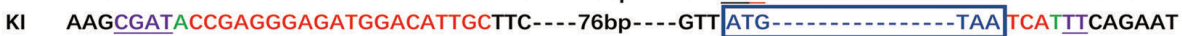

Figure 1. Generation of pF9 knockout (KO) pigs and hF9 knockin (KI) pigs. (A) Schematic diagram of the strategy used to generate the F9 KO pigs. Two sgRNAs were used to target the 5' untranslated region (UTR) and exon 1 (E1) of pF9 to promote DNA breaks. (A) The primer pair F1/R1 was used to identify the knockout of pF9. (B) The targeting regions were amplified using genomic DNA derived from the piglets. The gene is located on the sex chromosome: eight male piglets (F0-1, FO-2, FO-3, FO-6, FO-7, FO-8, FO-9, FO-10) presented single deletion bands, whereas the other four female piglets (FO-11, FO-12, FO-13, FO-14) who have two F9 alleles presented heterozygous bands. (C) The exact genotypes of pF9 KO pigs were determined by Sanger sequencing. The targeting sequences of the sgRNAs are marked in red, while the protospacer adjacent motifs (PAM) are marked in green. The mutated sequences of male pigs are presented above the line, and the corresponding sequences of female pigs are arranged below. KO-T1: the genotype of a 117 bp deletion; KO-T2: the genotype of a 118 bp deletion. (D) Strategy used for the CRISPR/Cas9-mediated knockin of $h F 9$ into the endogenous pF9 locus via homologous recombination. The donor DNA consisted of $h F 9$ coding sequences (CDS) and homologous porcine sequences (left arm and right arm), which were used to integrate hF9 into the porcine genome. 5' HA: 5' homologous arm (1.0 kb); 3' HA: 3' homologous arm (1.0 kb). (E) The newborn $h F 9 \mathrm{KI}$ piglets were identified via polymerase chain reaction using the primer pair F1/R1. (F) The genome sequences of $h F 9 \mathrm{KI}$ pigs indicate the successful insertion of the $h F 9 \mathrm{CDS}$. The modified bases that avoid recutting after homologous recombination are underlined and marked in purple. 
A

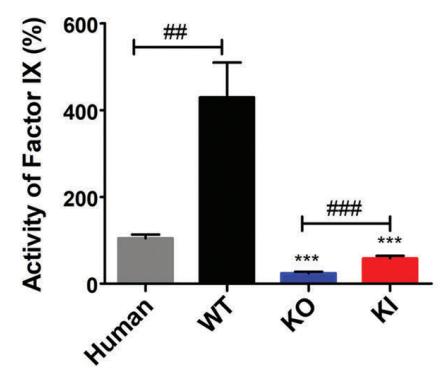

B

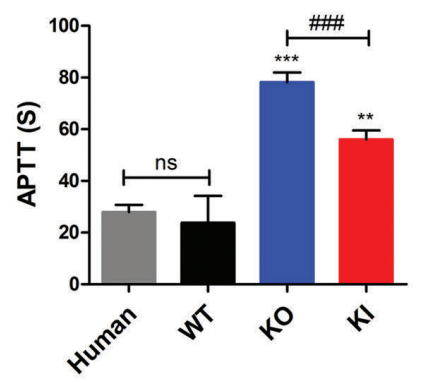

C

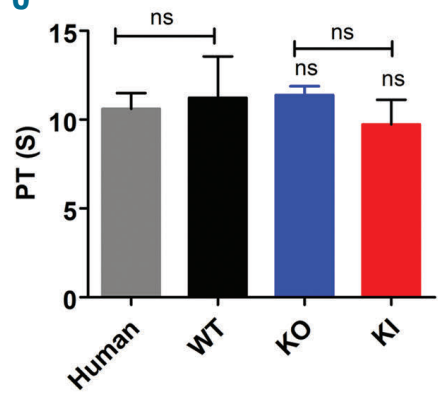

D

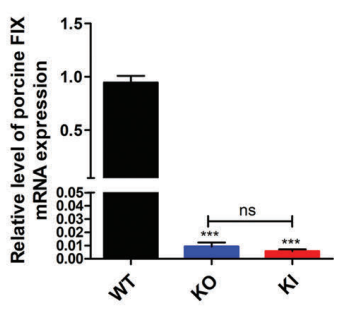

E

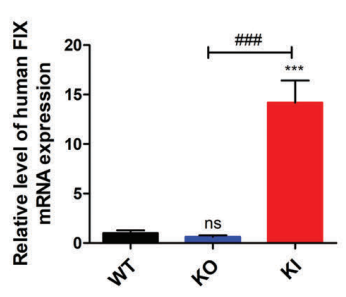

F

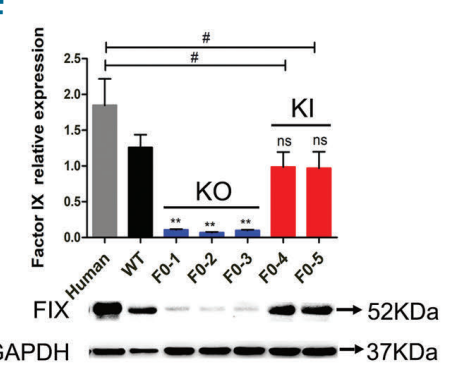

G

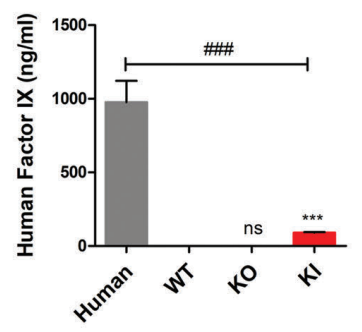

Figure 2. The analysis of blood coagulation and factor IX (FIX) levels. (A) FIX activities in the plasma of pigs and normal humans. The activity was calculated on the basis of a standard curve generated with normal human plasma and is expressed as a percentage of FIX activity in normal human plasma (FIX activity: WT: approx. 429\%; KO: approx. 23.8\%; KI: approx. 58\%). (B) Measurement of the APTT. (C) Measurement of the PT. (A-C: Human, n=3; WT, n=4; KO, n=4; KI, n=3). (D) quantitative-real-time-polymerase chain reaction (QRT-PCR) detection of mRNA expression levels of porcine factor IX in the livers from pF9 KO pigs and $h F 9$ KI pigs. (E) qRTPCR detection of mRNA expression levels of human factor IX in the livers from pF9 KO pigs and $h F 9$ KI pigs. (F) Western blotting of liver tissues with an antibody against human FIX and porcine FIX. GAPDH served as the control. Quantitation of the ratios of FIX to GAPDH is shown. Western blot analysis was repeated independently at least three times. (G) Determination of the FIX concentration in the plasma of pF9 KI pigs using human plasma as a control. The data are presented as the mean \pm standard deviation and were analyzed using an unpaired $t$-test with Welch's correction using GraphPad Prism software. The error bars represent the standard deviation. ${ }^{*} * P<0.005,{ }^{*} * * P<0.0001$, ns: not significant (vs. the WT pigs). ${ }^{*} P<0.05,{ }^{\# *} P<0.005,{ }^{* \# \#} P<0.0001$.

S3). Similarly, the positive cell clones were mixed as donor cells for SCNT; the detailed records are shown in Online Supplementary Table S2. Genotyping by PCR and sequencing analysis confirmed that the five delivered pigs were genetically identical and the $h F 9$ CDS had been inserted correctly (Figure $1 \mathrm{E}$ and F and Online Supplementary Figure S4). The results indicated that the $p F q \mathrm{KO}$ pigs and $h F 9$ knockin (KI) pigs developed successfully.

\section{Blood analysis of the pF9 knockout pigs and human F9 knockin pigs}

Clinically, as an X-linked recessive disorder, HB is common in men. Thus, we focused on male pigs to investigate their pathological and behavioral changes. Four $p F 9 \mathrm{KO}$ pigs, four WT pigs, and three $h F 9 \mathrm{KI}$ pigs were included in the blood analysis. FIX activity was severely decreased in $p F 9 \mathrm{KO}$ pigs, while it was partly increased in $h F 9 \mathrm{KI}$ pigs compared with the activity in $p F 9 \mathrm{KO}$ pigs (Figure $2 \mathrm{~A}$ ). When FIX activity in porcine plasma was expressed as the percentage of coagulation factor activity in WT pig plasma, it was approximately $5.5 \%$ (range: $4.5-6.2 \%$ ) in $\mathrm{pF} 9$ $\mathrm{KO}$ pigs and $13.5 \%$ (range: $12.3-15.0 \%$ ) in $h F 9 \mathrm{KI}$ pigs. Furthermore, $\mathrm{pF} 9 \mathrm{KO}$ pigs had a significantly prolonged activated partial thromboplastin time (APTT) compared with that of WT pigs, and the insertion of hF9 CDS partly ameliorated the condition (Figure $2 \mathrm{~B}$ ). There was no difference among the different groups with regard to the prothrombin time (PT) (Figure 2C). Apart from this, two of the $p F 9 \mathrm{KO}$ pigs had significantly decreased red blood cell (RBC) counts and hemoglobin ( $\mathrm{Hb}$ ) levels, as well as lower levels of total proteins and albumin (Table 1). Higher white blood cell (WBC) counts were found in both $p F 9$ $\mathrm{KO}$ pigs and $h F 9 \mathrm{KI}$ pigs. There were no significant differences in platelet counts among all groups.

Porcine FIX mRNA was extremely low in all $p F 9 \mathrm{KO}$ pigs and $h F 9 \mathrm{KI}$ pigs, and human FIX mRNA was effectively transcribed in $h F 9 \mathrm{KI}$ pigs (Figure $2 \mathrm{D}$ and $\mathrm{E}$ ). FIX protein synthesis in livers also decreased significantly in pF9 $\mathrm{KO}$ pigs, and human FIX could be synthesized in $h F 9$ KI pigs (Figure 2F). Human FIX could be successfully secreted into the blood of $h F 9 \mathrm{KI}$ pigs, approximately $9 \%$ (approx. 90.4 $\mathrm{ng} / \mathrm{mL}$ ) of the FIX level in normal human plasma (Figure $2 \mathrm{G}$ ). Based on the above analysis, the $p F 9 \mathrm{KO}$ pigs showed lower FIX expression and exhibited coagulation abnormalities. The insertion of $h F 9$ partly alleviated the abnor$\mathrm{mal}$ coagulation function in the $\mathrm{pF} 9 \mathrm{KO}$ pigs.

\section{Clinical observations of the porcine F9 knockout pigs and human F9 knockin pigs}

All piglets were separated from their mothers after delivery, and each piglet was housed individually by artificial suckling in a cage the insides of which had cushioned buffers to protect the piglets and avoid trauma (Figure 3A). Clinical observations of ten cloned pigs, including five $p F 9$ $\mathrm{KO}$ pigs, three $h F 9 \mathrm{KI}$ pigs, and two WT pigs were performed from birth to 8 weeks of age. No bleeding episodes were recorded in WT pigs throughout the observation period. All $p F 9 \mathrm{KO}$ pigs had multiple spontaneous bleeding episodes, while spontaneous bleeding with a lower frequency occurred in $67 \%$ of $h F 9$ KI pigs (2 of 3); no bleeding episode was found in one hFg KI pig (Online Supplementary Table S3). Most of the bleeding occurred in 
A

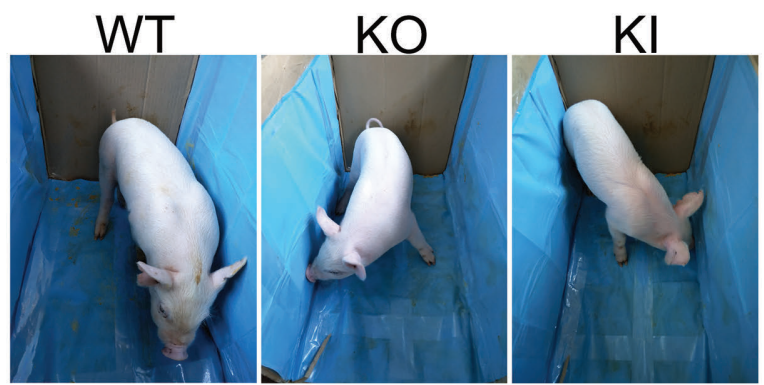

B
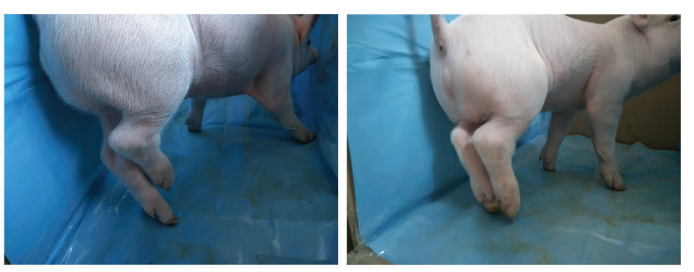

C

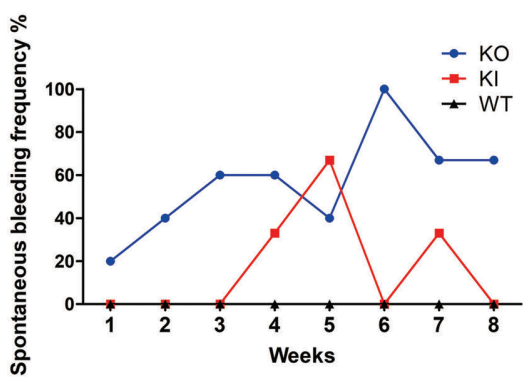

D
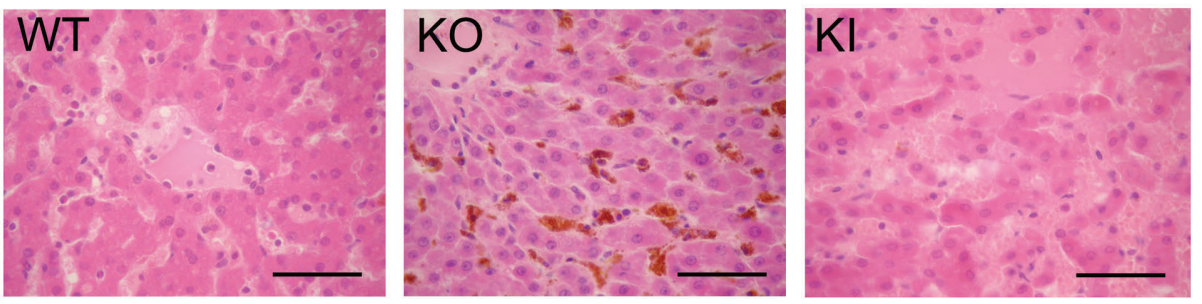

Figure 3. The bleeding phenotype of pF9 knockout (KO) pigs and hF9 knockin (KI) pigs. (A) Photographs of a pF9 KO piglet, an hF9 KI piglet and a wild-type (WT) piglet twenty days after birth. (B) Pictures illustrating joint swelling and limping in hemophilia B pigs. (C) Relative spontaneous bleeding frequency in pigs from birth to the age of 8 weeks with a peak at approximately $5-6$ weeks of age (WT, n=2; KO, n=5; KI, n=3). (D) Histological changes in porcine liver tissues. Scale bars: $50 \mu \mathrm{m}$.

Table 1. Some hematologic and serum biochemical parameters among the three groups.

\begin{tabular}{lccc} 
Parameter & WT pigs $(n=4)$ & KO pigs $(n=4)$ & Kl pigs $(n=3)$ \\
$\mathrm{RBC}, \mathrm{x} 10^{12} / \mathrm{L}$ & $6.15(4.8-7.5)$ & $3.55(2.1-5.2)$ & $4.67(3.8-6)$ \\
$\mathrm{WBC}, \mathrm{x} 0^{9} / \mathrm{L}$ & $24.55(20.8-27.7)$ & $37.95(32.1-44.9)$ & $30.5(24.4-35.5)$ \\
\hline $\mathrm{Plt}, \mathrm{x} 10^{9} / \mathrm{L}$ & $237.22(196.6-276)$ & $255.8(224.4-282.7)$ & $254.9(246.9-265.6)$ \\
$\mathrm{Hb}, \mathrm{g} / \mathrm{dL}$ & $13.23(10.7-16.7)$ & $9.23(6.9-11.6)$ & $10.6(9.2-12.1)$ \\
\hline Total protein, $\mathrm{g} / \mathrm{L}$ & $58.48(54.9-63.5)$ & $38.95(22.6-56.2)$ & $53.87(42.6-60.2)$ \\
Albumin, $\mathrm{g} / \mathrm{dL}$ & $2.90(2.65-3.21)$ & $2.02(1.2-2.85)$ & $2.69(2.03-3.06)$ \\
\hline
\end{tabular}

Numbers in parentheses represent the minimum to maximum values.WT:wild-type; KO: knockout; KI: knockin; n: number; RBC: red blood cell count;WBC: white blood cell count; Plt: platelet count; Hb: hemoglobin.

joints, especially the ankle joints (KO: 4 of 5 ; KI: 2 of 3 ) and the knee joints (KO: 4 of 5; KI: 0 of 3), which caused the pigs to limp (Figure $3 \mathrm{~B}$ ). Other joints, such as the elbow joints (KO: 2 of 5; KI: 1 of 3 ) and toe joints (KO: 1 of 5; KI: 1 of 3), also showed occasional bleeding. In addition, some bleeds were observed in other locations, including muscles (KO: 2 of 5; $\mathrm{KI}: 0$ of 3), nose (KO: 2 of 5 ; KI: 0 of 3), and eyes (KO: 1 of 5; KI: 0 of 3 ). Bleeds were less common during the first week after birth, and the peak frequency of bleeding occurred at 5-6 weeks of age (Figure 3C). Among the whole population, two pF9 KO pigs died of visceral bleeding, indicating that the spontaneous bleeding frequency may have been slightly higher than our observations. In addition, histological analysis of the livers of $p F q \mathrm{KO}$ pigs demonstrated erythrocyte destruction in the hepatic sinus and hemosiderin deposition; there were no significant differences between $h F 9 \mathrm{KI}$ pigs and WT pigs (Figure 3D). According to the abovedescribed clinical observations, $p F 9 \mathrm{KO}$ pigs exhibited serious coagulation disorders, and the bleeding episodes were ameliorated in $h F 9$ KI pigs.

Histological and radiological evaluation of arthropathic changes in porcine F9 knockout pigs and human F9 knockin pigs

Hemophilic arthropathy is characterized by two main 
features: chronic synovitis and cartilage destruction. An enlarged synovial membrane accompanied by inflammatory infiltrations was observed in $75 \%$ (3 of 4 ) of the $p F 9$ KO pigs, and a thin synovial membrane with subsynovial adipose tissue was seen in $75 \%$ ( 3 of 4 ) of the $h F 9 \mathrm{KI}$ pigs (Figure 4A). In addition, severe cartilage destruction was observed in $75 \%$ (3 of 4) of the pF9 KO pigs, while mild chondrocyte and proteoglycan losses were also found in $75 \%$ (3 of 4 ) of the $h F 9$ KI pigs (Figure 4B). The arthropathy score was applied to the histological analysis. There was a significant difference in synovial change between $p F 9 \mathrm{KO}$ pigs and $h F 9 \mathrm{KI}$ pigs and almost no signs of syn-

A
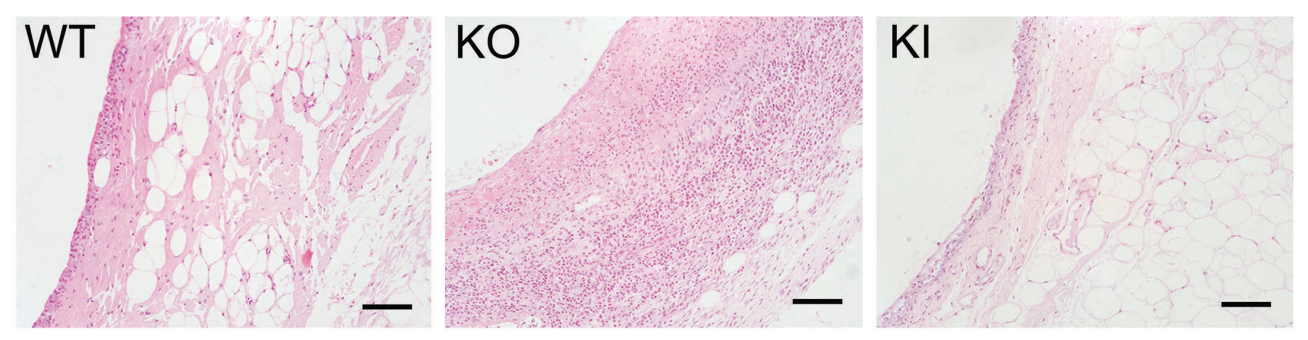

B
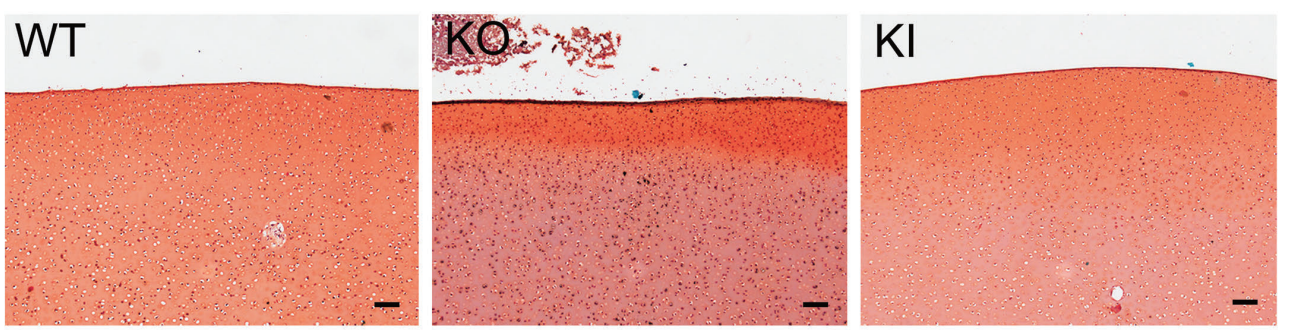

C

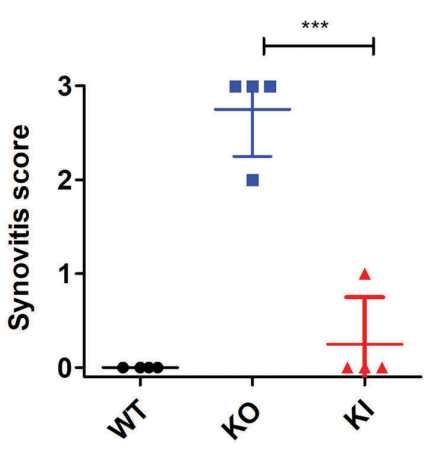

D

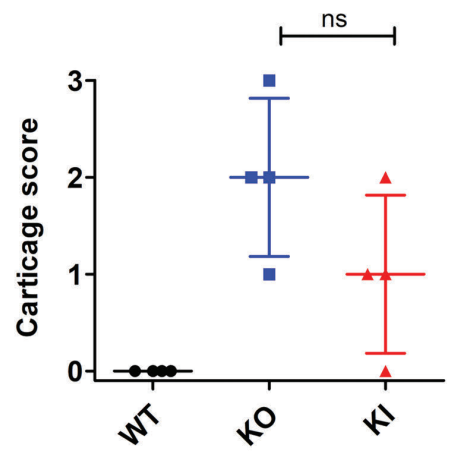

E
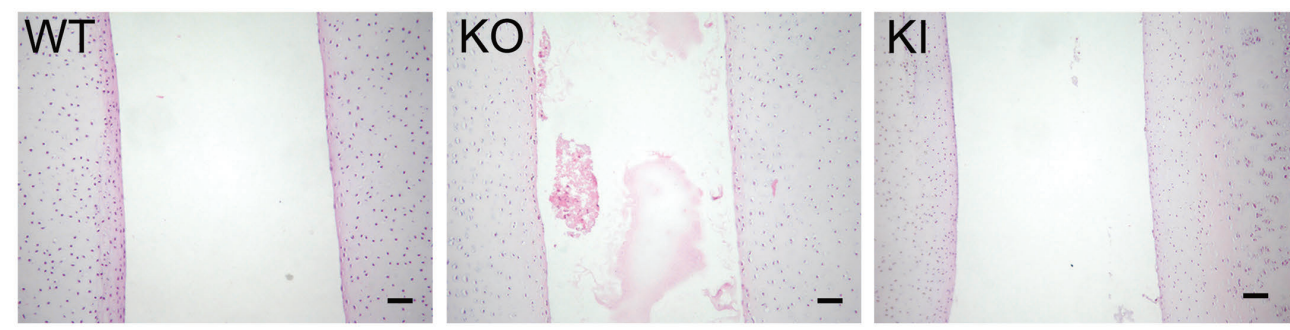

$\mathbf{F}$
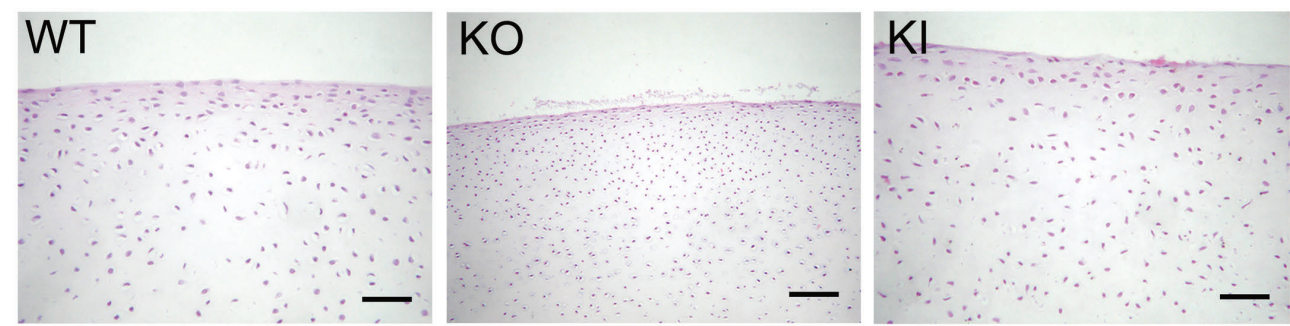

Figure 4. Histological analysis of the ankle joints of pF9 knockout (KO) pigs and $h F 9$ knockin (KI) pigs. (A) An enlarged and inflamed synovial membrane was shown in the $p F 9 \mathrm{KO}$ group, while the thin synovial membrane with subsynovial adipose tissue in the $h F 9 \mathrm{KI}$ group was comparable to that in the WT group. (B) Safranin 0 staining was performed to show proteoglycan loss and chondrocyte apoptosis in $p F 9$ KO pigs and $h F 9$ KI pigs. (C) Histological sections were scored to assess synovitis (0-3). (D) Histological sections were scored to assess cartilage degradation (O-3). (E) pF9 KO pigs exhibited coagulated blood in the ankle joint cavity. (F) Joint surface of the ankle joints. WT, $\mathrm{n}=4 ; \mathrm{KO}, \mathrm{n}=4 ; \mathrm{KI}, \mathrm{n}=4$. Scale bars: $50 \mu \mathrm{m}$. $* * * P<0.0001$, ns: not significant. 
ovitis were found in $h F 9 \mathrm{KI}$ pigs (Figure 4C). Cartilage destruction occurred in both $p F q \mathrm{KO}$ pigs and $h F 9 \mathrm{KI}$ pigs (Figure 4D). Apart from these findings, coagulated blood was present in the joint cavity of $p F 9 \mathrm{KO}$ pigs, creating a damaged microenvironment around the joint surfaces (Figure 4E and F).

To assess the impact of hemarthrosis on bone changes, the pigs were evaluated radiographically. Compared with those of WT pigs and $h F 9$ KI pigs, the epiphyseal plates of some joints in $\mathrm{pF} 9$ pigs were fused early (Figure 5A-C). Histological and radiological evaluation of arthropathic changes revealed that the arthropathic characteristics in $p F 9 \mathrm{KO}$ pigs were similar to the findings in joints of humans with hemophilic arthropathy.

\section{Discussion}

In this study, we generated an $\mathrm{HB}$ translational pig model and tested the possibility of $\mathrm{HB}$ therapy by CRISPR/Cas9-mediated gene insertion. This large mammalian model of $\mathrm{HB}$ showed characteristics similar to those of hemophilic patients. Most importantly, the hemophilic pigs had a high frequency of spontaneous bleeding episodes (especially in the ankles and knees), making these animals a useful translational model for studying hemophilic arthropathy in hemophiliac individuals. Compared with hemophiliac pigs, the transgenic pigs with a gene insertion of human F9 exhibited significantly ameliorated bleeding symptoms. This finding highlights the potential to replace the defective gene by gene insertion in situ.

First, while designing the target sites, we found that multiple point mutations in the F9 gene were present in the $5^{\prime}$ untranslated region (5' UTR) and signal peptide region based on clinically reported cases of $\mathrm{HB} .{ }^{30-33}$ The resulting missense mutations in people with hemophilia indicates FIX:C or FIX:Ag are less than 1\%; this corresponds to severe hemophilia, such as that found with point mutations occurring at $-55,+8$ and +19 , which are related to the initiation codon (Factor IX Variant Database). Therefore, we designed two sgRNAs to target partial sequences of the 5' UTR and signal peptide region to produce an $\mathrm{HB}$ pig model. In designing the donor vector for homologous recombination, except for the coding sequence of $h F 9$, the regulatory sequences preceding the endogenous initiation codon of $p F q$ were complemented to avoid affecting the expression of human FIX.

The mRNA and protein expression results in $\mathrm{HB}$ pigs showed that the expression of porcine FIX was extremely weak and that the APTT was approximately four times longer. When FIX activity in porcine plasma was expressed as the percentage of coagulation factor activity in WT pig plasma, it was approximately $5.5 \%$ (range: $4.5-$ $6.2 \%$ ) in pFq $\mathrm{KO}$ pigs and $13.5 \%$ (range: $12.3-15.0 \%$ ) in $h F 9 \mathrm{KI}$ pigs. Increasing plasma FIX levels as low as $1 \%$ results in the restoration of clotting activity. ${ }^{34-36}$ During the whole observation period, the frequency of spontaneous bleeding decreased markedly in hF9 KI pigs. However, there is still some way to go to achieve our ultimate objective. For patients, achieving relief from bleeding is not sufficient. Arthropathy develops progressively and this development is not interrupted once the initial hemarthrosis has started. In our models, there were some differences in paraclinical parameters among the three groups. We suspected that the decreases in $\mathrm{RBC}$ counts, $\mathrm{Hb}$, total protein and albumin levels in two $\mathrm{pF} 9 \mathrm{KO}$ pigs may be associated with chronic ongoing non-overt bleeding. The higher WBC counts in $p F q \mathrm{KO}$ pigs and $h F 9 \mathrm{KI}$ pigs could not be readily explained because they are not commonly seen in human hemophilic patients without acute infections. During an 8-week observation period, spontaneous bleeds occurred very frequently in the joints of $p F 9 \mathrm{KO}$ pigs. This is a unique feature that was not found in other animal models of hemophilia. The histological examination of the ankle joints of $p F 9 \mathrm{KO}$ pigs showed that without any intervention chronic proliferative synovitis and cartilage destruction occurred within two months. Hemophilic arthropathy is a multifactorial event, and detailed knowledge of the sequential cell and tissue responses after
A
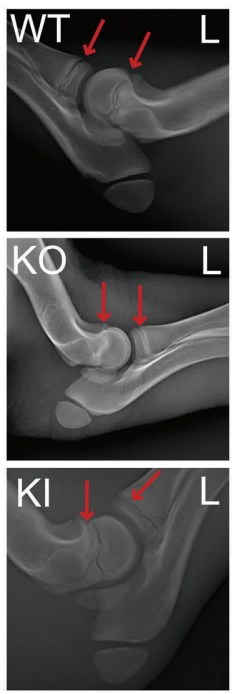
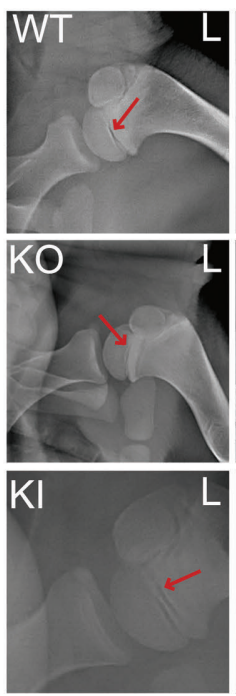

C
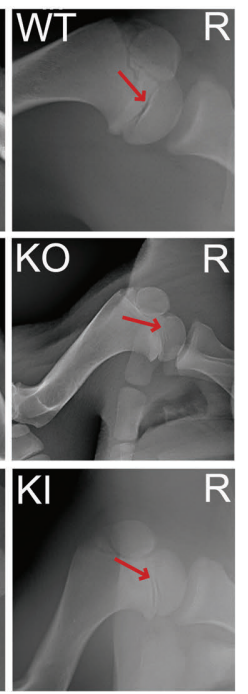
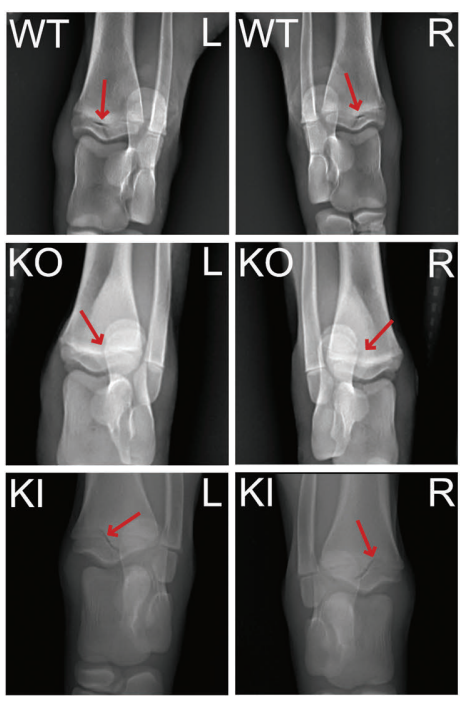

Figure 5. Radiological changes in some joints of pF9 knockout (KO) pigs and hF9 knockin (KI) pigs. (A) Elbow joints, (B) shoulder joints, (C) ankle joints. Arrows indicate the epiphyseal plates that were fused in hemophilic pigs. WT: wild-type; L: left; R: right. 
hemarthrosis is important for an in-depth understanding of the pathological process initiated on extensive bleeding into a joint. The hemophilic pig is a suitable disease model for such studies. As a large animal model, pigs pose many challenges. It takes a long time to obtain enough individuals, and a significant research investment is required to develop species-appropriate reagents, assays and expertise.

Site-specific insertion of $h F 9$ was achieved by using CRISPR/Cas9, which can break double-stranded DNAs to facilitate homologous recombination. The insertion of $h F 9$ alleviated clotting disorders in hemophiliac pigs, suggesting that it is feasible to replace defective genes with normal genes in situ. However, the gene replacement in our study occurred at the donor cell stage, not by directly completing gene correction using gene editing for individuals who are hemophiliac. In a recent report, researchers successfully corrected the bleeding phenotype in newborn and adult factor IX KO mice through in vivo gene editing mediated by CRISPR/Cas $9 .{ }^{37}$ The study provides convincing evidence of efficacy following in vivo genome editing in hemophilia and identifies the following points that should be considered in our next study: (i) application of the hyperactive FIX Padua variant; (ii) development of a recombinant vector suitable for HB patients with any mutations; (iii) a vector capable of effectively delivering the gene editing system; and (iv) the efficacy of Cas9-mediated in vivo genetic correction in newborn and adult individuals. Furthermore, the induction of neutralizing antibody (inhibitor) to the therapeutic protein sometimes precludes stable phenotypic correction follow- ing gene therapy, and the immune responses triggered by the functional protein, the gene editing system and the delivery vectors should be monitored over a long period of time. ${ }^{38-40}$

In conclusion, our study offers an alternative $\mathrm{HB}$ model for exploring the pathological process of hemophilic arthropathy and provides a possibility for the permanent correction of hemophilia in the future by genome editing in situ.

\section{Disclosures}

No conflicts of interest to disclose.

\section{Contributions}

$J H C, B Y A, B Y, X H P$ and $H M Y$ carried out the experimental work, the data collection and interpretation. $Q B Y, L Y W, X W Z$ and $H W$ participated in the coordination of experimental work. $X C T, H S O$ and DXP participated in the study design. JHC carried out the analysis and interpretation of data and drafted the manuscript. TTY, XDZ and XC provided the technical supports.

\section{Acknowledgments}

The authors would like to thank Tingting Yu and Xue Chen for their valuable technical contribution and Hongsheng Ouyang and Daxin Pang for helpful discussions. This work was supported by grants from the National Natural Science Foundation of China (Nos. 31572345 and 31472053) and the Jilin Scientific and Technological Development Program of China (No. 20170623035TC).

\section{References}

1. Jin NZ, Gopinath SCB. Potential blood clotting factors and anticoagulants. Biomed Pharmacother. 2016;84:356-365.

2. White GC 2nd, Rosendaal F, Aledort LM, et al. Definitions in hemophilia. Recommendation of the scientific subcommittee on factor VIII and factor IX of the scientific and standardization committee of the International Society on Thrombosis and Haemostasis. Thromb Haemost. 2001; 85(3):560.

3. Lillicrap D. FIX It in one go: enhanced factor IX gene therapy for hemophilia B. Cell. 2017:171(7):1478-1480

4. Dolan G, Benson G, Duffy A, et al. Haemophilia B: where are we now and what does the future hold? Blood Rev. 2018; 32(1):52-60.

5. Roosendaal G, Lafeber FP. Blood-induced joint damage in hemophilia. Semin Thromb Hemost. 2003;29(01):37-42.

6. Bolton-Maggs PH, Pasi KJ. Haemophilias A and B. Lancet. 2003:361(9371):1801-1809.

7. Lu W, Zhou Q, Yang H, et al. Gene therapy for hemophilia B mice with scAAV8-LP1hFIX. Front Med. 2016;10(2):212-218.

8. Nichols TC, Whitford MH, Arruda VR, Stedman HH, Kay MA, High KA. Translational data from adeno-associated virus-mediated gene therapy of hemophilia B in dogs. Hum Gene Ther Clin Dev. 2015; 26(1):5-14.

9. George LA, Sullivan SK, Giermasz A, et al. Hemophilia B gene therapy with a high-specific-activity factor IX variant. N Engl J Med. 2017;377(23):2215-2227.

10. Nathwani AC, Tuddenham EG, Rangarajan $\mathrm{S}$, et al. Adenovirus-associated virus vectormediated gene transfer in hemophilia B. N
Engl J Med. 2011;365(25):2357-2365.

11. Miesbach W, Meijer K, Coppens M, et al. Gene therapy with adeno-associated virus vector 5-human factor IX in adults with hemophilia B. Blood. 2018;131(9):10221031.

12. Nathwani AC, Reiss UM, Tuddenham EG, et al. Long-term safety and efficacy of factor IX gene therapy in hemophilia B. N Engl J Med. 2014;371(21):1994-2004.

13. Savic N, Schwank G. Advances in therapeutic CRISPR/Cas9 genome editing. Transl Res. 2016;168:15-21.

14.Lozier JN, Nichols TC. Animal models of hemophilia and related bleeding disorders. Semin Hematol. 2013;50(2):175-184.

15. Sabatino DE, Nichols TC, Merricks E, Bellinger DA, Herzog RW, Monahan PE. Animal models of hemophilia. Prog Mol Biol Transl Sci. 2012;105:151-209.

16. Monahan PE. The expanding menagerie: animal models of hemophilia A. J Thromb Haemost. 2010;8(11):2469-2471.

17.Pastoft AE, Lykkesfeldt J, Ezban M, Tranholm M, Whinna HC, Lauritzen B. A sensitive venous bleeding model in haemophilia A mice: effects of two recombinant FVIII products (N8 and Advate(R)). Haemophilia. 2012;18(5):782-788.

18. Elm T, Karpf DM, Ovlisen K, et al. Pharmacokinetics and pharmacodynamics of a new recombinant FVIII (N8) in haemophilia A mice. Haemophilia. 2012; 18(1):139-145.

19. Valentino LA, Hakobyan N, Kazarian T, Jabbar KJ, Jabbar AA. Experimental haemophilic synovitis: rationale and development of a murine model of human factor VIII deficiency. Haemophilia. 2004;10(3): 280-287.

20. Valentino LA, Hakobyan N. Histological changes in murine haemophilic synovitis: a quantitative grading system to assess bloodinduced synovitis. Haemophilia. 2006;12(6): 654-662.

21. Callan MB, Haskins ME, Wang P, Zhou S, High KA, Arruda VR. Successful phenotype improvement following gene therapy for severe hemophilia A in privately owned dogs. PLoS One. 2016;11(3):e0151800.

22. French RA, Samelson-Jones BJ, Niemeyer GP, et al. Complete correction of hemophilia B phenotype by FIX-Padua skeletal muscle gene therapy in an inhibitor-prone dog model. Blood Adv. 2018;2(5):505-508.

23. Cantore A, Ranzani M, Bartholomae CC, et al. Liver-directed lentiviral gene therapy in a dog model of hemophilia B. Sci Transl Med. 2015;7(277):277ra28.

24.Bendixen E, Danielsen M, Larsen K, Bendixen C. Advances in porcine genomics and proteomics-a toolbox for developing the pig as a model organism for molecular biomedical research. Brief Funct Genomics. 2010;9(3):208-219.

25. Ryu J, Prather RS, Lee K. Use of gene-editing technology to introduce targeted modifications in pigs. J Anim Sci Biotechnol. 2018; 9:5.

26.Perleberg C, Kind A, Schnieke A. Genetically engineered pigs as models for human disease. Dis Model Mech. 2018;11(1).

27. Cong L, Ran FA, Cox D, et al. Multiplex Genome engineering using CRISPR/cas systems. Science. 2013;339(6121):819-823.

28.Lai L, Kolber-Simonds D, Park KW, et al. Production of alpha-1,3-galactosyltransferase knockout pigs by nuclear transfer cloning. Science. 2002;295(5557):1089-1092.

29. Sorensen KR, Roepstorff K, Wiinberg B, et al. The F8(-/-) rat as a model of hemophilic arthropathy. J Thromb Haemost. 2016; 
14(6):1216-1225.

30. Heit JA, Thorland EC, Ketterling RP, et al. Germline mutations in Peruvian patients with hemophilia B: pattern of mutation in AmerIndians is similar to the putative endogenous germline pattern. Hum Mutat. 1998;11(5):372-376.

31.Ludwig M, Grimm T, Brackmann HH, Olek $K$. Parental origin of factor IX gene mutations, and their distribution in the gene. Am J Hum Genet. 1992;50(1):164-173.

32.Poon MC, Anand S, Fraser BM, Hoar DI, Sinclair GD. Hemophilia B carrier determination based on family-specific mutation detection by DNA single-strand conformation analysis. J Lab Clin Med. 1993;122 (1):55-63.
33.Wulff K, Bykowska K, Lopaciuk S, Herrmann FH. Molecular analysis of hemophilia B in Poland: 12 novel mutations of the factor IX gene. Acta Biochim Pol. 1999, 46(3):721-726.

34. Guan Y, Ma Y, Li O, et al. CRISPR/Cas9mediated somatic correction of a novel coagulator factor IX gene mutation ameliorates hemophilia in mouse. EMBO Mol Med. 2016;8(5):477-488.

35. High KA. Gene therapy for hemophilia: the clot thickens. Hum Gene Ther. 2014; 25(11):915-922.

36. Nathwani AC, Davidoff AM, Tuddenham EGD. Advances in gene therapy for hemophilia. Hum Gene Ther. 2017;28(11):10041012.
37. Wang L, Yang Y, Breton CA, et al CRISPR/Cas9-mediated in vivo gene targeting corrects hemostasis in newborn and adult factor IX-knockout mice. Blood. 2019;133(26):2745-2752.

38. VandenDriessche T, Collen D, Chuah MK. Gene therapy for the hemophilias. J Thromb Haemost. 2003;1(7):1550-1558.

39. Xu X, Wan T, Xin $\mathrm{H}$, et al. Delivery of CRISPR/Cas9 for therapeutic genome editing. J Gene Med. 2019;21(7):e3107.

40.Wagner DL, Amini L, Wendering DJ, et al High prevalence of Streptococcus pyogenes Cas9-reactive $\mathrm{T}$ cells within the adult human population. Nat Med. 2019;25(2): 242-248. 